\title{
Pregnancy outcomes following in vitro fertilization using fresh or frozen embryo transfer
}

\author{
Mahvash Zargar ${ }^{1}$, Sorour Dehdashti ${ }^{1}$, Mahin Najafian ${ }^{1}$, Parastoo Moradi Choghakabodi ${ }^{2}$ \\ ${ }^{1}$ Department of Obstetrics and Gynecology; Fertility, Infertility and Perinatology Research Center, Ahvaz \\ Jundishapur University of Medical Sciences, Ahvaz, Iran \\ ${ }^{2}$ Ahvaz Jundishapur University of Medical Sciences; Thalassemia \& Hemoglobinopathy Research Center, Health \\ Research Institute, Ahvaz, Iran
}

\begin{abstract}
Objective: The aim of this study was to investigate the pregnancy outcomes in women undergoing IVF using fresh and/or frozen embryo transfer.

Methods: In this retrospective patient record study, we reviewed 2,872 infertile women's files, who were candidates for IVF. The patients were classified into two groups, including those who underwent fresh embryo transfer $(n=1628)$ and/or frozen embryo transfer (FET) $(n=1244)$.

Results: Fertility was achieved in 313 (19.23\%) and $356(28.62 \%)$ patients, who underwent fresh ET and FET, respectively. The rates of clinical pregnancy, ongoing pregnancy, and live births were significantly higher in the FET group than the fresh ET group. The incidence of multiple pregnancies, perinatal mortality, abortion in the first trimester, preterm delivery, and low birth weight were significantly higher among fresh ET group [38 $(35.51 \%), 15(14.50 \%), 72(23.01 \%), 26(8.30 \%)$, and $33(10.54 \%)$, respectively] than in the FET group [25 $(15.33 \%), 6(6.87 \%), 63(17.69 \%), 14(3.93 \%)$, and 20 $(5.61 \%) ; p<0.05]$. In addition, the incidence of ectopic pregnancies, abortion in the second trimester, gestational diabetes, preeclampsia, and placenta previa were higher in the fresh ET group, but not significantly so $(p>0.05)$.

Conclusions: Women who underwent IVF via FET showed more successful fertility and pregnancy outcomes compared to those who underwent IVF by fresh ET.
\end{abstract}

Keywords: fresh embryo transfer, frozen embryo transfer, pregnancy, in vitro fertilization

\section{INTRODUCTION}

Infertility refers to the failure to achieve pregnancy after one year of unprotected sexual intercourse (Pelkonen et al., 2010; Dunkel-Schetter \& Lobel, 1991). Both female and male factors can cause infertility. Female and male factors are each solely responsible for $35 \%$ of infertility, and in $20 \%$ of cases a combination of female and male factors is involved; and in $10 \%$ of cases, there is no specific cause for the infertility found (Maheshwari et al., 2012; Roque et al., 2013; Lindsay \& Vitrikas, 2015). Other factors that increase the likelihood of infertility include environmental and occupational factors, the effects of toxins from tobacco use, strenuous exercise, very high or very low weight, and low age of couples (Lindsay \& Vitrikas, 2015; Pelkonen et al., 2010).

Successful fertility (successful embryo implantation) through the IVF method with fresh embryo is still low; cohort studies reported a chance of pregnancy between $18.9 \%$ and $41.8 \%$, with an average likelihood of $32 \%$ (Pelkonen et al., 2010). The IVF outcomes depend on a variety of factors, including maternal age, frozen or fresh embryo, donated eggs, etc. (Maheshwari et al., 2012). Considering that young age is one of the main pre-existing risk factors of ovarian hyperstimulation syndrome (OHSS), preservation of the frozen embryos for the next 3 to 5 years for ART cycle programs can help increase the chance of pregnancy and reduce the risk of multiple pregnancies (Roque et al., 2015; Dieamant et al., 2017).

Qualified embryos or fertilized eggs in IVF is one of the key parts of a successful pregnancy. In this regard, there is information to be checked, including the number of cells available for evaluation of fresh ET health and embryo quality grading (Weitzman et al., 2010). 48 hours after fertilization, we examined cell number, cell size and the fragmentation degree of cells. Whenever the number of fragmentation is greater, the embryo quality is lower and pregnancy rates may be lower. Three day-old embryo grading based on the number of cells, embryo fragmentation and symmetry or proportion of cells are divided into five grades E, D, C, B, A. Therefore, the highest IVF embryo viability has the lowest fragmentation and natural growth rate, usually including embryos in grades $\mathrm{B}, \mathrm{A}$ (Puissant et al., 1987). Embryo transfer is done in two ways: 1 . Fresh embryos fertilized at the same menstrual cycle. 2. Frozen embryos fertilized in previous cycles (Roque et al., 2013). The present study is aimed at comparing the pregnancy outcomes in women who underwent IVF using fresh or frozen embryo transfer.

\section{MATERIALS AND METHODS}

This retrospective study was carried out in 2019 after approval by the group council and the ethics committee of the Ahvaz Jundishapur University of Medical Sciences - Ahvaz, Iran (Ethical Code: IR.AJUMS.REC.1398.782). In total, 2,872 infertile women's files, who were referred to the Ahvaz Infertility Center and were candidates for ART, were reviewed and evaluated.

Patients with incomplete information and egg donation cases were excluded from the study. The patients were classified into two groups including those who underwent fresh ET $(n=1628)$ and/or frozen embryo transfer (FET) $(n=1244)$.

The method and process of these measures were in accordance with global and central protocols. Thus, all cases were followed up via a phone call. Pregnancy outcomes such as spontaneous abortion, preterm delivery, gestational diabetes, gestational hypertension, prenatal mortality, and other pregnancy consequences were assessed and recorded. In addition, the study continued until the end of the pregnancy for pregnant patients who were undergoing IVF.

Pregnancy using human chorionic gonadotropin (HCG) levels was performed on the $11^{\text {th }}$ day of embryo transfer, and clinical pregnancy was confirmed based on confirming 
an embryo heart rate at the $7^{\text {th }}$ week of gestation. Ongoing pregnancy was considered as the continuation of the pregnancy process after the $12^{\text {th }}$ week of gestation. Preterm delivery was considered as delivery before the $37^{\text {th }}$ week of gestation. Mortality in the prenatal period included stillborn infant deliveries or infants who were born alive from the $22^{\text {nd }}$ week of gestation and died on the $7^{\text {th }}$ day of birth. Other parameters such as multiple births, ectopic pregnancy, gestational diabetes, placenta previa, abortion in the first trimester, abortion in the second trimester, and low birth weight were extracted from the files and compared between the two groups.

\section{Statistical analysis}

Categorical variables were compared using the Chisquare test and presented as frequency and percentages while continuous variables were compared with independent t-test, and presented as the mean. Analysis of all results was performed by the SPSS software version 22 , and a $p$-value lower than 0.05 was considered statistically significant.

\section{RESULTS}

Reviewing the 2,872 files of infertile female candidates for IVF showed that fertility was achieved in 313 patients $(19.23 \%)$ and 356 patients $(28.62 \%)$ from the fresh ET group and the FET group, respectively. Statistically, the studied patients did not differ significantly in terms of maternal and paternal average age, type of infertility (primary or secondary), and the cause of infertility $(p>0.05)$ (Table 1).

In the fresh embryo and the frozen embryo groups, clinical pregnancy was respectively confirmed among the 111 cases $(35.46 \%)$ and 169 cases $(47.47 \%)$, which was significantly different $(p=0.0001)$. The ongoing pregnancy rate was significantly higher in the frozen embryo group $(p=0.0001)$. In addition, the live birth rate was significantly higher in the frozen embryo group [138 $(38.76 \%)]$, compared with the fresh embryo group [49 (15.65\%); $p=0.0001]$.

The incidence of multiple pregnancies, perinatal mortality, abortion in the first trimester, preterm delivery, and low birth weight $<2500 \mathrm{~g}$ were significantly higher in the fresh ET group than the FET group $(p<0.05)$. Although the incidence of ectopic pregnancies, abortion in the second trimester, gestational diabetes, preeclampsia, and placenta previa were higher in the fresh ET group, no statistically significant differences were found between the two groups $(p>0.05)$. The mean birth weight was significantly higher in the FET group $(p=0.001)$.
Causes of prenatal mortality were also investigated. The most important maternal cause of prenatal deaths in both fresh and frozen embryo groups was multiple pregnancies [( $34.18 \%$ vs. $20.51 \%$, respectively); Table 2$]$.

From a total of 356 FET, 294 cases $(82.58 \%$ ) had previously experienced a pregnancy failure following the first fresh ET, while 62 cases $(17.41 \%)$ had an early successful pregnancy. Subsequently, among these 62 cases, 38 and 24 cases showed successful pregnancies and live birth in frozen cycles, respectively. However, from 294 cases with a history of pregnancy failure, 128 cases became pregnant and 104 cases achieved a live birth.

Based on an intragroup statistical comparison between both subgroups of FET cases (with and/or without a history of early pregnancy), the clinical pregnancy rate was significantly higher in cases with a history of successful early pregnancy $(61.30 \%$ vs. $43.53 \% ; p=0.0174)$. However, the live birth rate was not significantly different between the two subgroups $[p=0.66$; Table 2]. All cases in the fresh ET group were in the first ET experience.

\section{DISCUSSION}

According to medical reports, IVF experts do not agree on which is better, fresh embryo transfer or frozen embryo transfer. Therefore, the present study aimed to investigate the outcomes of pregnancy following IVF with fresh and/or frozen embryos on 669 patients.

In Wang et al. (2017), the rate of ongoing pregnancy in the frozen embryo group (52\%) was significantly higher than in the fresh embryo group (45.3\%). They concluded that the frozen embryo protocol significantly increased the rate of implantation and pregnancy success compared to the fresh embryo. The results of Roque et al. (2015) showed that the probability of fertility among FET and fresh ET groups was $46.4 \%$ and $35.9 \%$, respectively, and the results of IVF in cases undergoing frozen ET were significantly better than fresh ET group. Our study showed that the rate of clinically confirmed pregnancies and the ongoing pregnancy in the frozen embryo group were significantly higher than in the fresh embryo group, which is consistent with the mentioned reports. Based on a study in Canada, the pregnancy success rate was about 33\% for fresh embryos, while it was reported as $24 \%$ for frozen embryos (Gunby et al., 2010), which was in contradiction with our findings.

According to Spijkers et al. (2017), the rate of preterm delivery (less than 37 weeks) was higher in the fresh embryo group. The results from Maheshwari et al. study (2012) showed that the rate of preterm delivery was lower in women who were fertilized using frozen embryos

\begin{tabular}{|c|c|c|c|c|}
\hline Variable & & $\begin{array}{l}\text { Fresh embryo } \\
(n=\mathbf{3 1 3})\end{array}$ & $\begin{array}{c}\text { Frozen embryo } \\
(n=356)\end{array}$ & $p$-value \\
\hline \multirow{2}{*}{ Age (Mean) } & Female & 34.73 & 33.34 & 0.81 \\
\hline & Male & 39.04 & 37.83 & 0.72 \\
\hline \multirow{2}{*}{ Types of infertility } & Primary & $224(71.57)$ & $248(69.66)$ & \multirow{2}{*}{0.41} \\
\hline & Secondary & $89(28.43)$ & $108(30.34)$ & \\
\hline \multirow{4}{*}{ Infertility causes } & Male factor & $167(53.35)$ & $191(53.65)$ & \multirow{4}{*}{0.83} \\
\hline & PCO & $102(32.59)$ & $111(31.18)$ & \\
\hline & Male factor/PCO & $32(10.22)$ & $38(10.67)$ & \\
\hline & Other causes & $44(14.06)$ & $54(15.17)$ & \\
\hline
\end{tabular}

Data are expressed as mean or number (\%).

Chi-Square test.

$p<0.05$ is considered as significant level. 
Table 2. Determining and comparing the consequences of pregnancy in two groups.

\begin{tabular}{|c|c|c|c|c|c|}
\hline \multicolumn{2}{|l|}{ Variables } & $\begin{array}{c}\text { Fresh embryo } \\
(n=313)\end{array}$ & \multicolumn{2}{|c|}{$\begin{array}{c}\text { Frozen embryo } \\
(n=356)\end{array}$} & $p$-value \\
\hline \multicolumn{2}{|c|}{ Clinical pregnancy } & $111(35.46 \%)$ & \multicolumn{2}{|c|}{$169(47.47 \%)$} & $0.0001 * * *$ \\
\hline \multicolumn{2}{|c|}{ Ongoing pregnancy } & $77(24.60 \%)$ & \multicolumn{2}{|c|}{$141(39.60 \%)$} & $0.0001 * * *$ \\
\hline \multicolumn{2}{|c|}{ Live birth rate } & $49(15.65 \%)$ & \multicolumn{2}{|c|}{$138(38.76 \%)$} & $0.0001 * * *$ \\
\hline \multicolumn{2}{|c|}{ Ectopic pregnancy } & $13(4.15 \%)$ & \multicolumn{2}{|c|}{$5(1.40 \%)$} & 0.62 \\
\hline \multirow{2}{*}{ Abortion } & $\begin{array}{l}\text { First-trimester } \\
\text { loss }\end{array}$ & $72(23.01 \%)$ & \multicolumn{2}{|c|}{$63(17.69 \%)$} & $0.022 *$ \\
\hline & $\begin{array}{l}\text { Second-trimester } \\
\text { loss }\end{array}$ & $10(11.60 \%)$ & \multicolumn{2}{|c|}{$9(6.87 \%)$} & 0.64 \\
\hline \multicolumn{2}{|l|}{ Twain } & $38(35.51 \%)$ & \multicolumn{2}{|c|}{$25(15.33 \%)$} & $0.024^{*}$ \\
\hline \multicolumn{2}{|c|}{ Gestational diabetes } & $17(24.64 \%)$ & \multicolumn{2}{|c|}{$31(23.66 \%)$} & 0.132 \\
\hline \multicolumn{2}{|c|}{ Placenta Previa } & $16(5.11 \%)$ & \multicolumn{2}{|c|}{$17(4.77 \%)$} & 0.859 \\
\hline \multicolumn{2}{|c|}{ Preeclampsia } & $24(7.66 \%)$ & \multicolumn{2}{|c|}{$26(7.30 \%)$} & 0.88 \\
\hline \multicolumn{2}{|c|}{ Preterm delivery } & $26(8.30 \%)$ & \multicolumn{2}{|c|}{$14(3.93 \%)$} & $0.021 *$ \\
\hline \multicolumn{2}{|c|}{ Low birth weight $<2500 \mathrm{~g}$} & $33(10.54 \%)$ & \multicolumn{2}{|c|}{$20(5.61 \%)$} & $0.021 *$ \\
\hline \multicolumn{2}{|c|}{ Birth weight (kg) } & $2.8 \pm 1.03$ & \multicolumn{2}{|c|}{$3.2 \pm 1.8$} & $0.001 *$ \\
\hline \multicolumn{2}{|c|}{ Perinatal mortality } & $15(14.50 \%)$ & \multicolumn{2}{|c|}{$6(6.87 \%)$} & $0.0032 *$ \\
\hline & & & $\begin{array}{l}\text { FET cases with } \\
\text { successful } \\
\text { early pregnancy } \\
\text { history }(n=62)\end{array}$ & $\begin{array}{c}\text { FET cases } \\
\text { with failed } \\
\text { pregnancy } \\
\text { history }(n=294) \\
\end{array}$ & \\
\hline \multicolumn{3}{|c|}{ Clinical pregnancy after FET cycles } & $38(61.30 \%)$ & $128(43.53 \%)$ & $0.011 *$ \\
\hline \multicolumn{3}{|c|}{ Live birth rate after FET cycles } & $24(38.70)$ & $104(35.37)$ & 0.66 \\
\hline
\end{tabular}

Data are expressed as number (\%).

Fisher's exact test and/or Chi-Square.

FET: Frozen embryo transfer

$* p<0.05$ is considered as significant level.

(Maheshwari et al., 2012). The results of Spijkers et al. (2017) showed that the birth weight was higher in the frozen embryo group than in the fresh embryo group. The results of Luke et al. (2017) reported a higher risk of weight gain in the frozen embryo group compared to a fresh embryo in both the first and second pregnancies. Our findings have also confirmed these points, in which case the preterm delivery rate was higher in the fresh ET group, while the mean birth weight was significantly higher in the FET. In addition, the number of low birth weight infants in the fresh embryo group was significantly higher.

The results of Pelkonen et al. (2010) showed that the chance of low birth weight in infants born by FET was lower than in infants born from fresh embryo. In addition, the average birth weight for infants born by FET was 134 grams higher than in infants born from fresh embryos. In Maheshwari et al. (2012), low birth weight among infants born from fresh embryo was more evident than in FET infants.

In the present study, although the prevalence of patients with preeclampsia was higher in the fresh embryo group than in the FET group, this difference was not statistically significant. This result was consistent with the results from Blazquez et al. (2018); they showed that despite the high prevalence of preeclampsia in pregnant women with IVF, frozen embryos did not make a considerable reduction in the risk of preeclampsia, its duration, and gestational hypertension.

In the present study, perinatal mortality was significantly higher in the fresh embryo group. Multiple pregnancies were the most important maternal cause of prenatal deaths in both fresh and frozen embryo groups.

Based on Zamani Kiasari et al. (2010), the prenatal mortality rate was reported to be $7.2 \%$ in infants born from normal pregnancies. Our results showed that the mortality rate during the prenatal period in patients undergoing FET was almost lower than those who were undergoing fresh embryo transfers. In this regard, our results are confirming the results from Kansal Kalra et al. (2011); they evaluated 368 IVF pregnancies, including 238 cases of fresh ET and 130 cases of FET. The perinatal morbidity, first-trimester loss, and other adverse outcomes were significantly higher in IVF pregnancies with fresh ET compared with FET pregnancies. In addition, their results showed that the multiple pregnancy rate in the fresh ET group was considerably higher than the FET group, i.e. FET was more likely to result in a singleton pregnancy (Kansal Kalra et al., 2011).

Pelkonen et al. (2010) reported that the likelihood of premature birth was lower in the frozen embryo group than in the fresh embryo group. Frozen embryos also had no adverse effects on prenatal outcomes compared to fresh embryos, and was even better in some cases. Based on Maheshwari et al. (2012), the likelihood of perinatal mortality in infants born from frozen embryos were lower. In our study, the multiple pregnancy and abortion rates in the first trimester were significantly higher in the fresh embryo group than in the FET group. In addition, the rate of ectopic pregnancies, gestational diabetes, placenta previa, and abortion in the second trimester were higher in the fresh embryo group, but not significantly so. A systematic 
and meta-analysis review study carried out by Roque et al. (2013) stated that the fertility results using IVF with frozen embryo were better than in the fresh embryo. They assumed that these results might be due to better access to synchrony between endometrium and embryo in the frozen embryo transfer method.

Our results regarding the effect of previous fresh cycles on FET cycles are in agreement with the findings reported by Ashrafi et al. (2011) and Bushaqer et al. (2020), but in contrast with the reports from Doherty et al. (2014) and Bdolah et al. (2015). Our results showed that the clinical pregnancy rate in cases of FET with a history of successful early pregnancy was significantly higher than cases with the failed pregnancy history (61.30\% vs. $43.53 \%)$, which means that the previous fresh cycle protocol may affect the pregnancy outcomes arisen from subsequent FET cycles. However, the previous fresh cycle outcomes did not affect the live birth rate, which confirms the results reported by El-Toukhy et al. (2003) and Bushaqer et al. (2020). Their results showed that embryo survival arisen from frozen cycle is not affected by the fresh cycles (Bushaqer et al. 2020; El-Toukhy et al., 2003). In this regard, some researchers theorized that the good quality embryos in fresh and frozen cycles might lead to pregnancy in both cycles. However, opposite researchers believed that the top quality embryos would be often chosen for fresh cycles, and so, less reproductive embryos are left for FET cycles.

\section{CONCLUSION}

Women who underwent IVF by frozen embryo transfer showed more successful fertility and pregnancy outcomes compared to those who underwent IVF by fresh embryo transfer.

\section{Study strength}

Although a few studies have previously showed a strong influence of frozen ET on the consequences of fertility and pregnancy, this study is one of the rare studies, which more comprehensively and accurately examined the effect of embryo quality on all consequent pregnancies and childbirth following IVF.

\section{ACKNOWLEDGMENTS}

This study was approved and supported by the Ahvaz Jundishapur University of Medical Sciences (Ethical Code: IR.AJUMS.REC.1398.782; Grant.No: FIRC-9817).

\section{CONFLICT OF INTEREST}

All authors report no conflicts of interest relevant to this article. However, Dr. Mahvash Zargar, First and correspondence author, has received a research grant from Ahvaz Jundishapur University of Medical Sciences, Ahvaz, Iran.

\section{Corresponding author:}

Mahvash Zargar

Department of Obstetrics and Gynecology

Fertility, Infertility and Perinatology Research Center

Ahvaz Jundishapur University of Medical Sciences

Ahvaz, Iran

Email: zargar_m@ajums.ac.ir

\section{REFERENCES}

Ashrafi M, Jahangiri N, Hassani F, Akhoond MR, Madani T. The factors affecting the outcome of frozen-thawed embryo transfer cycle. Taiwan J Obstet Gynecol. 2011;50:159-64. PMID: 21791301 DOI: 10.1016/j.tjog.2011.01.037
Bdolah $Y$, Zemet R, Aizenman E, Lossos F, Abram TB, Shufaro Y. Frozen-Thawed Embryo Transfer Success Rate is Affected by Age and Ovarian Response at Oocyte Aspiration Regardless of Blastomere Survival Rate. JBRA Assist Reprod. 2015;19:210-5. PMID: 27203194 DOI: 10.5935/1518-0557.20150041

Blazquez A, García D, Vassena R, Figueras F, Rodriguez A. Risk of pre-eclampsia after fresh or frozen embryo transfer in patients undergoing oocyte donation. Eur J Obstet Gynecol Reprod Biol. 2018;227:27-31. PMID: 29879546 DOI: $10.1016 /$ j.ejogrb.2018.05.030

Bushaqer NJ, Alkhudhairy NN, Alturaigi ZM, Alhamad RM, Mohawesh WA, Alraka FE, Ayyoub HA, Nawal MD. The effect of fresh IVF cycle characteristics on frozen embryo transfer (FET) outcomes. JBRA Assist Reprod. 2020;24:135-42. PMID: 32072802 DOI: 10.5935/15180557.20190074

Dieamant FC, Petersen CG, Mauri AL, Comar V, Mattila M, Vagnini LD, Renzi A, Petersen B, Nicoletti A, Oliveira JB, Baruffi RL, Franco JG Jr. Fresh embryos versus freeze-all embryos - transfer strategies: Nuances of a meta-analysis. JBRA Assist Reprod. 2017;21:260-72. PMID: 28837037 DOI: $10.5935 / 1518-0557.20170048$

Doherty LF, Martin JR, Kayisli U, Sakkas D, Patrizio P. Fresh transfer outcome predicts the success of a subsequent frozen transfer utilizing blastocysts of the same cohort. Reprod Biomed Online. 2014;28:204-8. PMID: 24365019 DOI: $10.1016 /$ j.rbmo.2013.09.030

Dunkel-Schetter C, Lobel M. Psychological Reactions to Infertility. In: Stanton AL, Dunkel-Schetter C, eds. Infertility. Perspectives from Stress and Coping Research. New York: Springer US; 1991. p. 29-57.

El-Toukhy T, Khalaf Y, Al-Darazi K, Andritsos V, Taylor A, Braude P. Effect of blastomere loss on the outcome of frozen embryo replacement cycles. Fertil Steril. 2003;79:1106-11. PMID: 12738503 DOI: 10.1016/s00150282(03)00072-4

Gunby J, Bissonnette F, Librach C, Cowan L; IVF Directors Group of the Canadian Fertility and Andrology Society. Assisted reproductive technologies (ART) in Canada: 2006 results from the Canadian ART Register. Fertil Steril. 2010;93:2189-201. PMID: 19439295 DOI: $10.1016 / j$. fertnstert.2009.03.102

Kansal Kalra S, Ratcliffe SJ, Milman L, Gracia CR, Coutifaris C, Barnhart KT. Perinatal morbidity after in vitro fertilization is lower with frozen embryo transfer. Fertil Steril. 2011;95:548-53. PMID: 20663500 DOI: 10.1016/j.fertnstert.2010.05.049

Lindsay TJ, Vitrikas KR. Evaluation and treatment of infertility. Am Fam Physician. 2015;91:308-14. PMID: 25822387

Luke B, Brown MB, Wantman E, Stern JE, Toner JP, Coddington CC 3rd. Increased risk of large-for-gestational age birthweight in singleton siblings conceived with in vitro fertilization in frozen versus fresh cycles. J Assist Reprod Genet. 2017;34:191-200. PMID: 27909843 DOI: 10.1007/ s10815-016-0850-x 
Maheshwari A, Pandey S, Shetty A, Hamilton M, Bhattacharya $S$. Obstetric and perinatal outcomes in singleton pregnancies resulting from the transfer of frozen thawed versus fresh embryos generated through in vitro fertilization treatment: a systematic review and meta-analysis. Fertil Steril. 2012;98:368-77.e1-9. PMID: 22698643 DOI: 10.1016/j.fertnstert.2012.05.019

Pelkonen S, Koivunen R, Gissler M, Nuojua-Huttunen S, Suikkari AM, Hydén-Granskog C, Martikainen $H$, Tiitinen A, Hartikainen AL. Perinatal outcome of children born after frozen and fresh embryo transfer: the Finnish cohort study 1995-2006. Hum Reprod. 2010;25:914-23. PMID: 20124395 DOI: 10.1093/humrep/dep477

Puissant F, Van Rysselberge M, Barlow P, Deweze J, Leroy $\mathrm{F}$. Embryo scoring as a prognostic tool in IVF treatment. Hum Reprod. 1987;2:705-8. PMID: 3437050 DOI: 10.1093/oxfordjournals. humrep.a136618

Roque M, Lattes K, Serra S, Solà I, Geber S, Carreras R, Checa MA. Fresh embryo transfer versus frozen embryo transfer in in vitro fertilization cycles: a systematic review and meta-analysis. Fertil Steril. 2013;99:156-62. PMID: 23040524 DOI: 10.1016/j.fertnstert.2012.09.003

Roque M, Valle M, Guimarães F, Sampaio M, Geber S. Freeze-all policy: fresh vs. frozen-thawed embryo transfer. Fertil Steril. 2015;103:1190-3. PMID: 25747130 DOI: 10.1016/j.fertnstert.2015.01.045
Spijkers S, Lens JW, Schats R, Lambalk CB. Fresh and Frozen-Thawed Embryo Transfer Compared to Natural Conception: Differences in Perinatal Outcome. Gynecol Obstet Invest. 2017;82:538-46. PMID: 28501865 DOI: $10.1159 / 000468935$

Wang A, Santistevan A, Hunter Cohn K, Copperman A, Nulsen J, Miller BT, Widra E, Westphal LM, Yurttas Beim $P$. Freeze-only versus fresh embryo transfer in a multicenter matched cohort study: contribution of progesterone and maternal age to success rates. Fertil Steril. 2017;108:254-61.e4. PMID: 28579411 DOI: 10.1016/j. fertnstert.2017.05.007

Weitzman VN, Schnee-Riesz J, Benadiva C, Nulsen J, Siano L, Maier D. Predictive value of embryo grading for embryos with known outcomes. Fertil Steril. 2010;93:658-62. PMID: 19410247 DOI: 10.1016/j. fertnstert.2009.02.032

Zamani Kiasari A, Kabirzadeh A, Mohseni Saravi B, Rezazadeh E, Khademlou M, Biaza T. Rate and Causes of Perinatal Mortality in Imam Hospital, Sari 2007. Iran J Obstet Gynecol Infertil. 2010;12:23-30. DOI: 10.22038/ IJOGI.2009.5892 